\title{
$4=$ \\ USO DE CALCULADORA: LEVANTAMENTO DE TRABALHOS PUBLICADOS NOS ENCONTROS MINEIROS DE EDUCAÇÃO MATEMÁTICA
}

\author{
USE OF CALCULATOR: COLLECTION OF PUBLISHED PAPERS IN THE \\ MATHEMATICAL EDUCATION MEETINGS FROM MINAS GERAIS STATE
}

Nayara Katherine Duarte Pinto ${ }^{1}$

Keli Cristina Conti

\begin{abstract}
RESUMO: Este trabalho apresenta dados e algumas análises do estudo documental sobre o uso de calculadoras nas aulas de Matemática, por meio dos Anais dos Encontros Mineiros de Educação Matemática (EMEM de 1997 a 2015) e fez parte de um projeto de Iniciação Científica. O estudo teve como objetivo sistematizar os trabalhos que possuíam foco no uso da calculadora na sala de aula e realizar algumas análises desses documentos e foi caracterizado como pesquisa bibliográfica ou estudo documental. Após defesa do uso da calculadora nas aulas de Matemática, descrevemos as fases do levantamento e organização dos dados e os resultados da pesquisa, e por fim, concluímos que enquanto divulgação de trabalhos realizados junto à estudantes ou de divulgação de pesquisa ainda temos um número bastante reduzido de trabalhos que focam a utilização da calculadora ou seu uso como ferramenta no desenvolvimento de atividades matemáticas em sala de aula.
\end{abstract}

PALAVRAS-CHAVES: Calculadora, Educação Matemática, Laboratório de Ensino de Matemática

\begin{abstract}
ABSTRAT:This article presents data and some analyzes of the documentary study on the use of calculators in Mathematics classes, through the Annals of the Encontros Mineiros de Educação Matemática (EMEM from 1997 to 2015) and is part of a Scientific Initiation project. The aim of the study was to systematize the work that had a focus on the use of the calculator in the classroom and accomplish some analysis of these documents and was characterized as bibliographic research or documentary study. After defending the use of the calculator in Mathematics classes, we describe the phases of the survey and organization of the data and the results of the research, and finally, we conclude that while the dissemination of work done with students or research dissemination we still have a very small number of article that focus on the use of the calculator or its use as a tool in the development of mathematical activities in the classroom.
\end{abstract}

\footnotetext{
${ }^{1}$ Mestranda em Educação e Docência pela Universidade Federal de Minas Geais

2 Professora Adjunta do Departamento de Métodos e Técnicas de Ensino da Faculdade de Educação Universidade Federal de Minas Gerais
}

Tangram - Revista de Educação Matemática, Dourados - MS - v.1, n. 3, pp. 64 - 80 (2018) ISSN: 2595-0967 
Uso de calculadora: levantamento de trabalhos publicados nos Encontros Mineiros de Educação Matemática

KEYWORDS: Calculator, Mathematics Education, Mathematics Teaching Laboratory.

\section{INTRODUÇÃO}

Esta pesquisa integra um projeto de Iniciação Científica intitulado "Contribuições do Laboratório de Ensino de Matemática para a formação inicial do professor que ensina Matemática" que visou ampliar o Laboratório de Ensino de Matemática (LEM) na Faculdade de Educação da Universidade Federal de Minas Gerais (FaE/UFMG). O projeto busca também analisar e interpretar práticas de formação e de atuação de futuros professores de forma a compreender e ressaltar a importância de um LEM para a formação inicial do professor que ensinará Matemática e seu reflexo no processo de ensino e aprendizagem dos estudantes.

Neste artigo apresentamos os dados do estudo documental sobre o uso de calculadoras nas aulas de Matemática, por meio dos Anais dos Encontros Mineiros de Educação Matemática (EMEM) e algumas análises desses documentos referentes ao tema pesquisado. As calculadoras foram escolhidas por fazerem parte do LEM e serem alvo de vários estudos e oficinas sobre sua utilização no âmbito do projeto citado, com foco na parte prática e buscando um contraponto com as pesquisas realizadas.

O EMEM foi escolhido, por ser, de acordo com a Sociedade Brasileira de Educação Matemática, Regional Minas Gerais (SBEM - MG), "atualmente, o maior evento acadêmico-científico na área de Educação Matemática de Minas Gerais, por reunir professores e pesquisadores de diversas instituições mineiras e também de outros estados". Até o presente momento ocorreram sete Encontros Mineiros de Educação Matemática que serão detalhados mais adiante.

A seguir discutiremos um pouco da importância do uso da calculadora nas aulas de Matemática e posteriormente apresentamos a pesquisa nos anais.

\section{O USO DE CALCULADORA NAS AULAS DE MATEMÁTICA}

Embora o uso cotidiano das calculadoras na sociedade seja frequente, ainda há certa resistência, quando mencionamos o seu uso no ambiente educacional.

Tangram - Revista de Educação Matemática, Dourados - MS - v.1, n. 3, pp. 64 - 80 (2018) ISSN: 2595-0967 
Já se discutia, na década de 1980, em Educação Matemática, o uso de computadores, calculadoras simples e científicas no ensino e aprendizagem da Matemática, de acordo com Borba, Silva e Gadanidis (2014). Posteriormente, o uso da calculadora foi recomendado pelos Parâmetros Curriculares Nacionais (PCN - Brasil, 1997) em várias situações, assim como o uso de computadores e outros materiais, destacando seu importante papel no processo de ensino e aprendizagem, considerando que o acesso a elementos como a calculadora e computadores, "já é uma realidade para parte significativa da população" (Brasil, 1997, p. 34).

O documento reforçava que os usos de calculadoras "precisam estar integrados a situações que levem ao exercício da análise e da reflexão, em última instância, a base da atividade matemática" (Brasil, 1997, p. 19) e também defendia seu uso como um instrumento que traria contribuições para o ensino de Matemática:

Estudos e experiências evidenciam que a calculadora é um instrumento que pode contribuir para a melhoria do ensino da Matemática. A justificativa para essa visão é o fato de que ela pode ser usada como um instrumento motivador na realização de tarefas exploratórias e de investigação (BRASIL, 1997, p. 34).

Em documento mais recente, a BNCC (Base Nacional Comum Curricular, Brasil, 2017), há diversas menções ao uso de calculadoras, desde os Anos Iniciais do Ensino Fundamental. Com foco nas competências de cálculo, o documento apresenta:

No que diz respeito ao cálculo, é necessário acrescentar, à realização dos algoritmos das operações, a habilidade de efetuar cálculos mentalmente, fazer estimativas, usar calculadora e, ainda, para decidir quando é apropriado usar um ou outro procedimento de cálculo (BRASIL, 2017, p. 274).

O documento também apresenta que os materiais tecnológicos, como as calculadoras, "precisam estar integrados a situações que levem à reflexão e à sistematização, para que se inicie um processo de formalização" (BRASIL, 2017, p. 274).

Nesse sentido, sobre as competências de cálculo, Lopes e Rodriguez (2009) enfatizam que se utilizada corretamente contribui para o desenvolvimento de competências de cálculo, que são de acordo com os autores: calculadora, estimativa, cálculo mental e cálculo escrito. Os autores apresentam a ilustração (Figura 1) de uma mesa para reforçar a importância do equilíbrio entre as competências. Cada "pé da mesa" representaria uma competência.

Tangram - Revista de Educação Matemática, Dourados - MS - v.1, n. 3, pp. 64 - 80 (2018) ISSN: 2595-0967 
Figura 1: Competências de cálculo

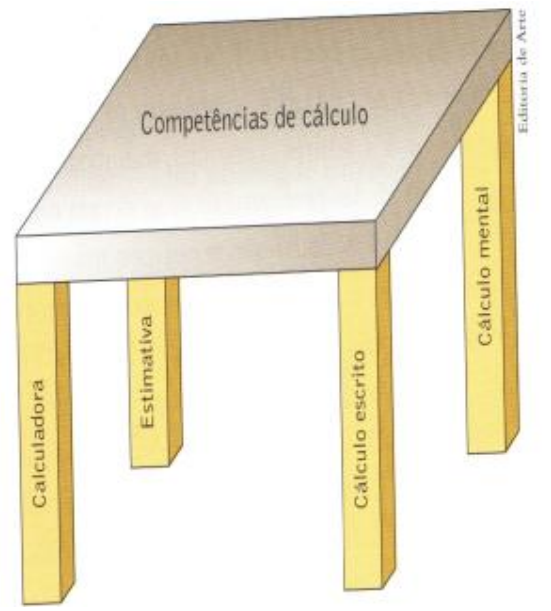

Fonte: Lopes; Rodriguez; 2009, p. 147.

De acordo com Lopes e Rodriguez (2009), boas propostas com o uso de calculadoras potencializariam "a capacidade dos alunos de fazer, mais e melhor, cálculo mental e estimativa, bem como ajudam a compreender o que fazem (às vezes mecanicamente) no cálculo escrito" (p. 147).

Lopes e Rodriguez (2009) também apresentam que uma justificativa antiga para não se usar a calculadora na escola era a do custo. Atualmente isso não se justifica, pois é possível adquirir uma calculadora simples "pelo preço de uma passagem de ônibus municipal" (p. 148).

Ainda, sobre o uso da calculadora, destacamos alguns mitos e medos, de acordo com Van de Walle (2009), em grande parte baseados na pouca informação a respeito:

a) Se as crianças usarem calculadoras, elas não aprenderão os "fundamentos";

b) As calculadoras tornam os estudantes preguiçosos;

c) Os estudantes devem aprender o "modo real" antes de usar calculadoras;

d) Os estudantes se tornarão dependentes demais de calculadoras. (VAN DE WALLE, 2009, p. 131-132)

Tangram - Revista de Educação Matemática, Dourados - MS - v.1, n. 3, pp. 64 - 80 (2018) ISSN: 2595-0967 
Uso de calculadora: levantamento de trabalhos publicados nos Encontros Mineiros de Educação Matemática

Concordamos com Van de Walle (2009), que "em vez de temer o dano potencial que as calculadoras podem causar, é importante compreender como as calculadoras podem contribuir para a aprendizagem da Matemática" (p. 130).

Consideramos que uma abordagem correta da calculadora pode subsidiar o desenvolvimento dos estudantes com as operações e o raciocínio lógico, bem como podem ser usadas para desenvolver conceitos e para fortalecer a resolução de problemas, são usadas nas práticas sociais e também podem economizar tempo (VAN DE WALLE, 2009). Para que uma calculadora encontre o resultado correto é necessário ser manipulada pelo ser humano, havendo um raciocínio antecipado para a realização das operações e nesse sentido “a calculadora será usada como recurso, não para substituir a construção de procedimentos de cálculo pelo aluno, mas para ajudá-lo a compreendê-los" (BRASIL, 1997, p. 45). Isso também é reforçado por Van de Walle (2009), ao expressar "quando usadas adequadamente, as calculadoras aumentam a aprendizagem; elas não atrapalham o caminho da aprendizagem" (p. 132) e também é expresso na BNCC, quando apresenta que recursos didáticos como as calculadoras (entre outros), "têm um papel essencial para a compreensão e utilização das noções matemáticas" (BRASIL, 2017, p. 274).

Conscientes da importância de se estimular o uso das calculadoras na sala de aula e da disseminação de pesquisas a respeito é que propusemos um estudo, no âmbito no nosso estado (MG), por meio dos Anais dos Encontros Mineiros de Educação Matemática e algumas análises destes documentos referentes ao tema pesquisado.

\section{DESENVOLVIMENTO DA PESQUISA}

No âmbito de uma Iniciação Científica, realizamos uma pesquisa bibliográfica ou estudo documental (FIORENTINI; LORENZATO, 2006). Como mencionamos anteriormente, objetivamos por meio da leitura e análise dos Anais do EMEMs sistematizar os trabalhos que possuíam foco no uso da calculadora na sala de aula e realizar algumas análises desses documentos.

Até o presente momento, como já mencionado, ocorreram sete Encontros Mineiros de Educação Matemática, sendo eles: o I EMEM realizado em dezembro de 1997 na cidade de Ouro Preto; o II EMEM e III EMEM ocorreram em Belo Horizonte, o segundo em dezembro de 2000 e o terceiro em novembro de 2003; o IV EMEM aconteceu em novembro

Tangram - Revista de Educação Matemática, Dourados - MS - v.1, n. 3, pp. 64 - 80 (2018) ISSN: 2595-0967 
Uso de calculadora: levantamento de trabalhos publicados nos Encontros Mineiros de Educação Matemática

de 2006 em Diamantina; o V EMEM foi realizado em novembro de 2009 em Lavras; e o VI EMEM aconteceu em novembro de 2012 em Juiz de Fora. O VII EMEM ocorreu em outubro de 2015 em São João Del Rei. Está previsto para o ano de 2018 o VIII EMEM.

De acordo com a Sociedade Brasileira de Educação Matemática, Regional Minas Gerais (SBEM - MG), o EMEM tem como principais objetivos:

- Promover o intercâmbio de conhecimentos na área de Educação Matemática;

- Divulgar os resultados de pesquisas realizadas em Minas Gerais;(...)

- Difundir os aportes que as novas tecnologias oferecem à Educação Matemática;

- Discutir o ensino e a aprendizagem da Matemática na sociedade atual;

- Aproximar as pesquisas acadêmicas das práticas educacionais escolares e estimular o intercâmbio de experiências de sala de aula nos distintos níveis de ensino. (SOCIEDADE BRASILEIRA E EDUCAÇÃO MATEMÁTICA - REGIONAL MINAS GERAIS, s.p)

Com a finalidade de atingir nosso objetivo optamos em dividir a pesquisa em quatro etapas, sendo elas: $\left.1 .^{a}\right)$ localizar os documentos dos anais dos EMEM;.$^{a}$ ) pesquisa inicial da palavra calculadora nos trabalhos de Relato de Experiência e Comunicação Científica; 3. a) pesquisa detalhada a partir da leitura dos títulos, resumos, palavra chaves e trechos dos textos que apareciam a palavra calculadora; 4. ${ }^{a}$ ) fichamentos de leituras detalhando e sistematizando dados dos trabalhos que possuíam foco no uso das calculadoras na sala. A partir deste momento passaremos a descrever cada etapa do desenvolvimento da pesquisa.

A primeira etapa foi localizar os anais dos EMEM e com o auxílio de parceiros conseguimos localizar seis anais, mas não tivemos acesso aos anais de I EMEM. A maioria dos anais eram arquivos em CDs, exceto o do II EMEM que conseguimos uma cópia impressa. A partir deste momento, segunda fase, optamos por pesquisar nas categorias de Relato de Experiência e Comunicação Científica, dos documentos que tivemos acesso, os textos referentes ao uso de calculadora nas aulas de Matemática. Logo após, para os documentos digitalizados utilizamos a ferramenta de pesquisa e digitamos a palavra "calculadora", já para o documento impresso fizemos a leitura completa dos textos para localizar se havia a palavra calculadora. Em seguida, criamos um fichamento de leitura inicial, para auxiliar nesta fase e ao encontrarmos algum artigo listamos o título, autores e categoria. As tabelas a seguir apresentam a contagem geral dos artigos que tinham a palavra pesquisada. Na tabela 1, nossa contagem foi realizada nas comunicações científicas (C.C.) e na tabela 2, nos relatos de experiência (R.E).

Tangram - Revista de Educação Matemática, Dourados - MS - v.1, n. 3, pp. 64 - 80 (2018) ISSN: 2595-0967 
Uso de calculadora: levantamento de trabalhos publicados nos Encontros Mineiros de Educação Matemática

Tabela 1: Tabela geral dos dados das Comunicações Científicas

\begin{tabular}{|c|c|c|c|}
\hline Evento & $\begin{array}{l}\text { Total de trabalhos } \\
\text { apresentado }\end{array}$ & $\begin{array}{l}\text { Quantidade de } \\
\text { trabalhos que } \\
\text { possuíam a palavra } \\
\text { calculadora }\end{array}$ & $\begin{array}{l}\text { Porcentagem de } \\
\text { trabalhos que } \\
\text { mencionam } \\
\text { calculadora em } \\
\text { relação ao total } \\
\text { da categoria }\end{array}$ \\
\hline II EMEM & 30 & 0 & $0 \%$ \\
\hline III EMEM & 41 & 6 & $14,6 \%$ \\
\hline IV EMEM & 39 & 1 & $2,5 \%$ \\
\hline V EMEM & 94 & 9 & $9,6 \%$ \\
\hline VI EMEM & 57 & 8 & $14,1 \%$ \\
\hline VII EMEM & 99 & 13 & $13,2 \%$ \\
\hline Total & 360 & 37 & $10,3 \%$ \\
\hline
\end{tabular}

Fonte: Elaborado pelas autoras (2018)

Tabela 2: Tabela geral dos dados dos Relatos de Experiências

\begin{tabular}{|c|c|c|c|}
\hline Evento & $\begin{array}{l}\text { Total de trabalhos } \\
\text { apresentado }\end{array}$ & $\begin{array}{l}\text { Quantidade de } \\
\text { trabalhos que } \\
\text { possuíam a palavra } \\
\text { calculadora }\end{array}$ & $\begin{array}{l}\text { Porcentagem de } \\
\text { trabalhos que } \\
\text { mencionam } \\
\text { calculadora em } \\
\text { relação ao total } \\
\text { da categoria }\end{array}$ \\
\hline
\end{tabular}

Tangram - Revista de Educação Matemática, Dourados - MS - v.1, n. 3, pp. 64 - 80 (2018) ISSN: 2595-0967 
Uso de calculadora: levantamento de trabalhos publicados nos Encontros Mineiros de Educação Matemática

$\begin{array}{llll}\text { II EMEM } & \text { Não se aplica } & \text { Não se aplica } & - \\ \text { III EMEM } & 56 & 2 & 3,6 \% \\ \text { IV EMEM } & 10 & 2 & 20 \% \\ \text { V EMEM } & 59 & 9 & 15,3 \% \\ \text { VI EMEM } & \text { Não se aplica } & \text { Não se aplica } & - \\ \text { VII EMEM } & \text { Não se aplica } & \text { Não se aplica } & - \\ \text { Total } & & & \mathbf{1 0 , 4 \%}\end{array}$

Fonte: Elaborado pelas autoras (2018)

Nos II, VI e VII EMEM não tiveram a categoria RE, por isso preenchemos estas células da tabela com o termo não se aplica. Deste modo, no II EMEM só ocorreram comunicações científicas e nenhuma delas referente à calculadora. Pelas informações das tabelas 1 e 2 temos um total de 13 trabalhos na categoria de Relato de Experiência e 37 trabalhos na categoria de Comunicação Científica, totalizando 50 publicações do II ao VII EMEM.

Gostaríamos de destacar que nessa primeira tabela foram incorporados os trabalhos que ao pesquisar a palavra "calculadora", foi encontrada ao menos uma vez. Na tabela a seguir apresenta uma segunda contagem dos dados após realizarmos a terceira etapa que consistiu em fazer a leitura dos títulos, resumos, palavra chaves e trechos dos textos que apareciam a palavra calculadora. Desta vez selecionamos apenas os trabalhos que tinham o foco no uso da calculadora.

Tabela 3: Tabela após a triagem dos dados

\begin{tabular}{llll}
\hline Evento Relato de Experiência & $\begin{array}{l}\text { Comunicação } \\
\text { Científica }\end{array}$ & Total
\end{tabular}

Tangram - Revista de Educação Matemática, Dourados - MS - v.1, n. 3, pp. 64 - 80 (2018) ISSN: 2595-0967 


$\begin{array}{llll}\text { II EMEM } & \text { Não se aplica } & 0 & 0 \\ \text { III EMEM } & 1 & 1 & 2 \\ \text { IV EMEM } & 1 & 0 & 1 \\ \text { V EMEM } & 2 & 0 & 2 \\ \text { VI EMEM } & \text { Não se aplica } & 1 & 1 \\ \text { VII EMEM } & \text { Não se aplica } & 1 & 1 \\ \text { Total } & \mathbf{4} & \mathbf{3} & \mathbf{7} \\ \end{array}$

Fonte: Elaborado pelas autoras (2018)

Ao compararmos as tabelas anteriores podemos notar que o número total de trabalhos na segunda tabela, sete, é menor em relação à soma dos trabalhos das duas primeiras tabelas, 50 trabalho, este fato se deve que muitos desses trabalhos citam a utilização de calculadora em algum momento do texto. Entretanto, os focos principais dos trabalhos eram, por exemplo, em softwares, tecnologias ou uso do computador. Uma parte significativa dos trabalhos a palavra calculadora estava apenas associado a material disponível para utilização ou que se era permitido o uso, já outros em citações bibliográficas. Encontramos também no trecho de duas Comunicações Científica do VII EMEM, menções como: não há a necessidade calculadora digitais em alguns processos e que a matemática era não apenas uma calculadora. Devido a isso, os trabalhos não foram considerados na etapa seguinte.

$\mathrm{Na}$ etapa seguinte, a quarta, criamos um fichamento de leitura detalhado, contendo título resumo e palavras chaves dos setes arquivos que selecionamos como os que abordavam o tema pesquisado como eixo central. A partir deste momento passamos a detalhar mais os dados em: nível de ensino pesquisado, tipo de instituição pesquisado e tipo de instituição de origem dos autores. Deste modo, optamos por organizar estes dados em gráficos.

No Gráfico 1 temos os dados da primeira classificação:

Tangram - Revista de Educação Matemática, Dourados - MS - v.1, n. 3, pp. 64 - 80 (2018) ISSN: 2595-0967 
Uso de calculadora: levantamento de trabalhos publicados nos Encontros Mineiros de Educação Matemática

Gráfico 1: Nível de ensino pesquisado

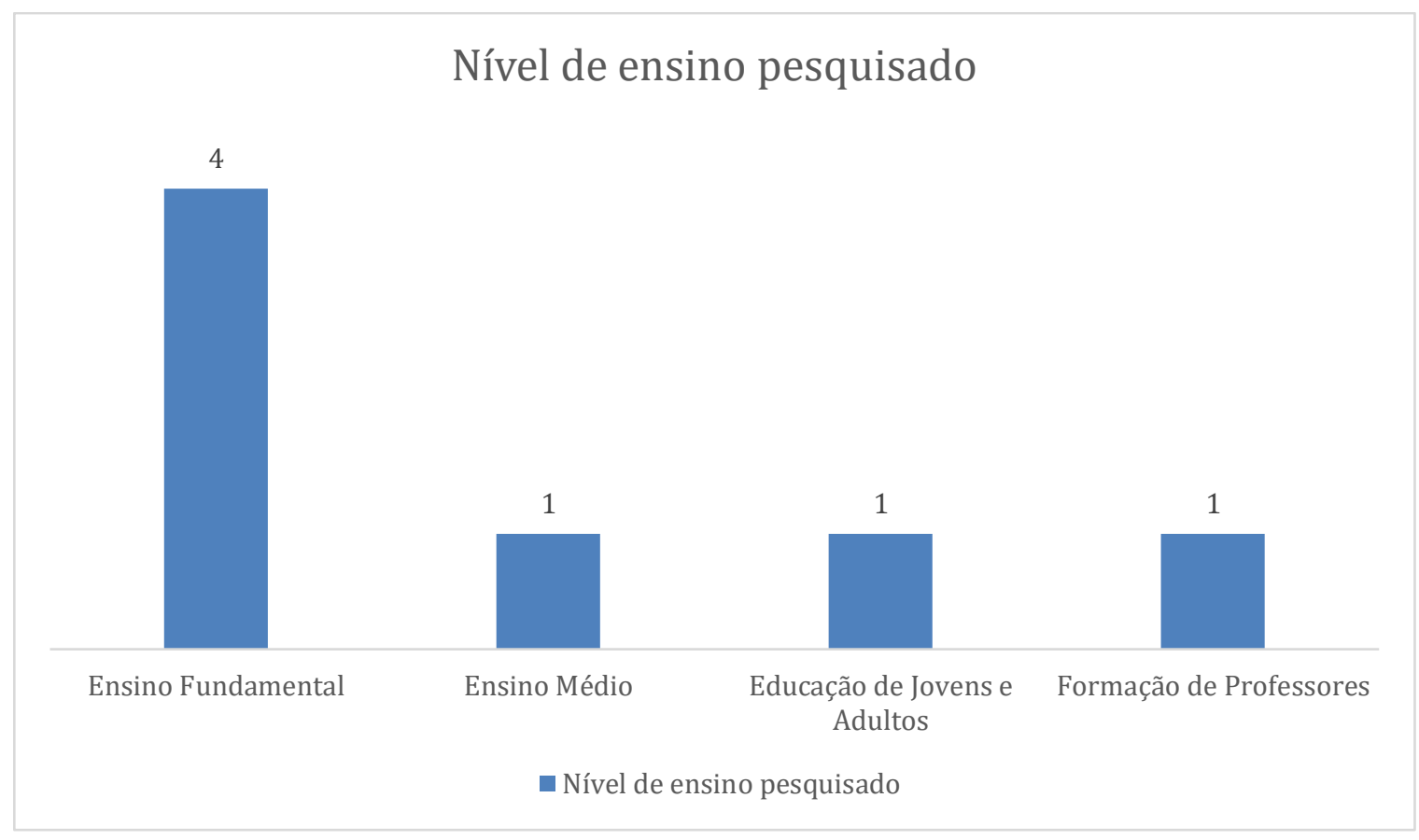

Fonte: Elaborado pelas autoras (2018)

Pelas informações do Gráfico 1 podemos notar que predominantemente (4 trabalhos) pesquisaram no âmbito do Ensino Fundamental, e um caso em cada um dos seguimentos do Ensino Médio, Educação de Jovens e Adultos e Formação de Professores. Desta forma, podemos dizer que o foco da maioria das pesquisas ocorrerem no Ensino Fundamental.

No gráfico 2 temos os dados do segundo detalhamento - tipo de instituição pesquisada:

Gráfico 2: Categoria da instituição que a pesquisa foi desenvolvida

Tangram - Revista de Educação Matemática, Dourados - MS - v.1, n. 3, pp. 64 - 80 (2018) ISSN: 2595-0967 
Uso de calculadora: levantamento de trabalhos publicados nos Encontros Mineiros de Educação Matemática

\section{Categoria da instituição que a pesquisa foi desenvolvida}

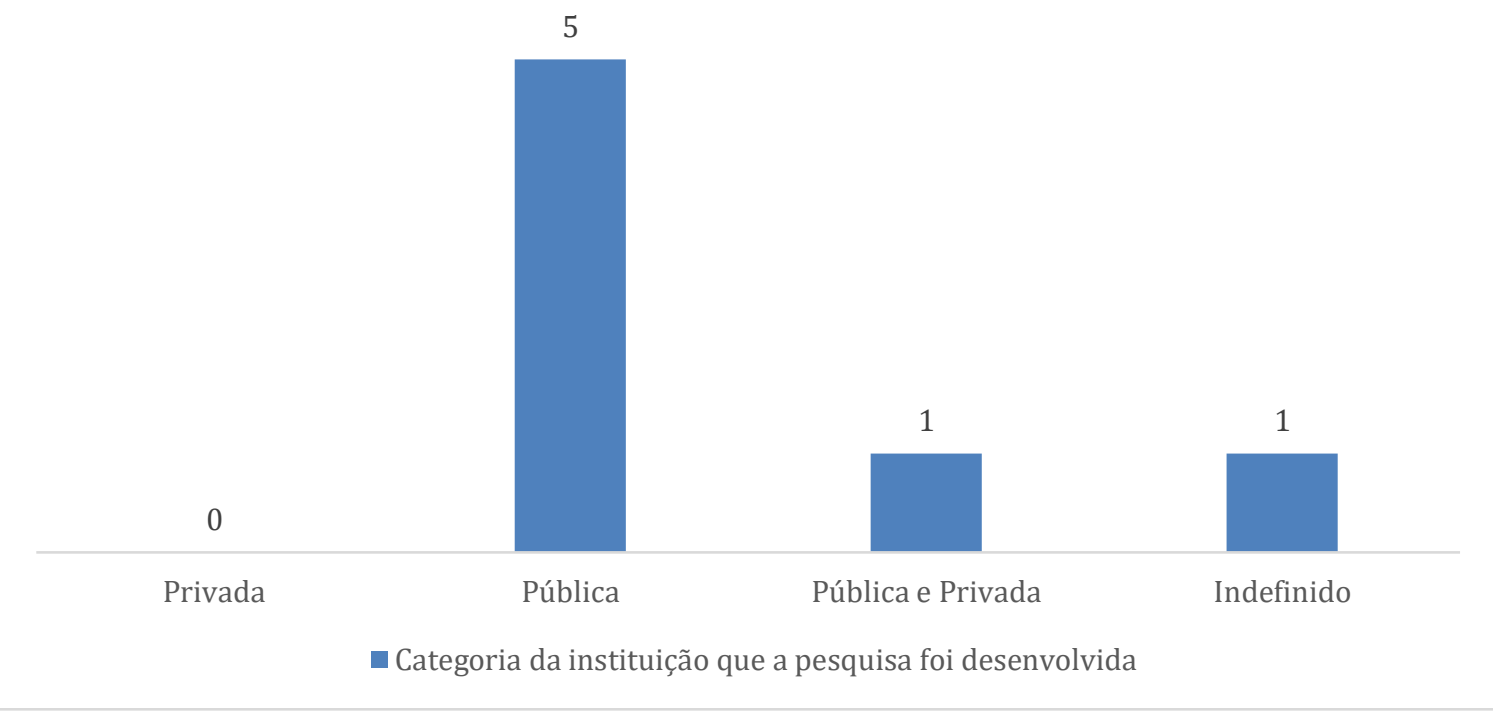

Fonte: Elaborado pelas autoras (2018)

Por meio do gráfico 2 podemos notar que as pesquisas não se desenvolveram em escolas privadas (ou particulares) exclusivamente, mas houve uma pesquisa que foi realizada simultaneamente em escolas públicas e privadas. Gostaríamos de destacar que o trabalho classificado como indefinido foi devido ao trabalho pesquisar livros didáticos e que em momento nenhum do texto citou onde seriam aplicadas as atividades desenvolvidas após as pesquisas. A categoria que houve mais classificações foi do ensino público com 5 trabalhos, e desses trabalhos, três casos no âmbito municipal, um caso no âmbito estadual e um no âmbito federal.

O gráfico a seguir apresenta os dados do último detalhamento: tipo da instituição de origem dos autores.

Gráfico 3: Categoria de instituição de origem dos autores

Tangram - Revista de Educação Matemática, Dourados - MS - v.1, n. 3, pp. 64 - 80 (2018) ISSN: 2595-0967 
Uso de calculadora: levantamento de trabalhos publicados nos Encontros Mineiros de Educação Matemática

\section{Categoria tipo de instituição de origem dos autores}

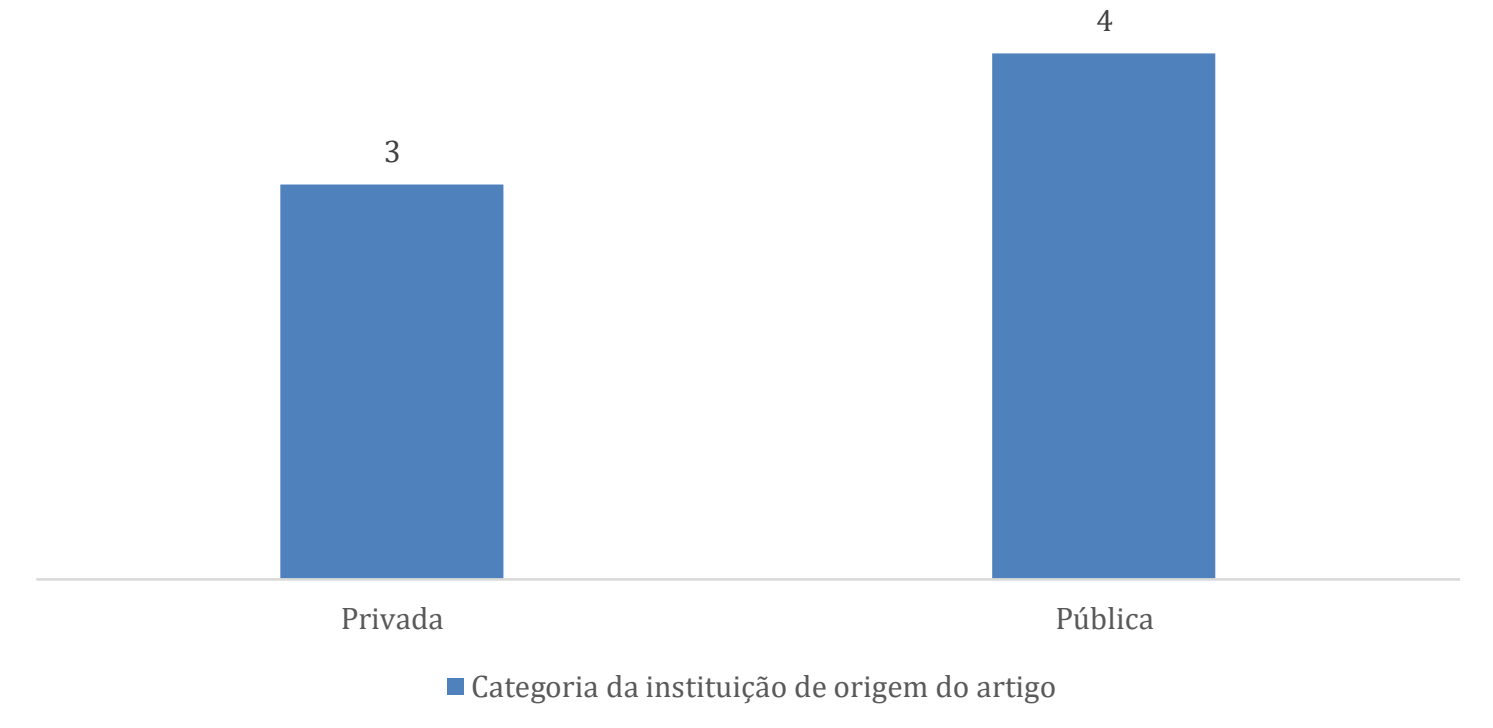

Fonte: Elaborado pelas autoras (2018)

Por meio do gráfico 3 podemos dizer que foi equilibrado os tipos das instituições de origem com três casos das instituições privadas e 4 de instituições públicas. Pelo fato de encontramos sete trabalhos, optamos por detalhar algumas informações dos mesmos. Elaboramos dois quadros, um para R.E. e outro de C.C. com os eventos, títulos, autores e palavras-chaves, quando houver, dos artigos contidos na pesquisa e algumas impressões que tivemos ao fazer a leitura dos textos.

Tangram - Revista de Educação Matemática, Dourados - MS - v.1, n. 3, pp. 64 - 80 (2018) ISSN: 2595-0967 
Quadro 1: Trabalhos da Categoria Relato de Experiência

\begin{tabular}{|l|l|l|l|}
\hline Evento & Título & Autores & Palavras-Chaves \\
\hline III EMEM & $\begin{array}{l}\text { A calculadora como recurso } \\
\text { didático no ensino } \\
\text { aprendizagem da } \\
\text { matemática: análise das } \\
\text { propostas apresentadas nos } \\
\text { livros didáticos do ensino } \\
\text { Aundamental de; MELO, } \\
\text { Maria Cristina } \\
\text { Rotsen de. }\end{array}$ & Não possui \\
\hline IV EMEM & $\begin{array}{l}\text { Utilização de calculadora na } \\
\text { EJA? }\end{array}$ & $\begin{array}{l}\text { FERREIRA, Ana } \\
\text { Rafaela }\end{array}$ & $\begin{array}{l}\text { Educação de Jovens } \\
\text { e Adultos, uso de } \\
\text { calculadora, aula de } \\
\text { matemática }\end{array}$ \\
\hline V EMEM & $\begin{array}{l}\text { Ensino de funções, limites e } \\
\text { continuidade em ambientes } \\
\text { educacionais informatizados } \\
\text { para cursos de introdução ao } \\
\text { cálculo: relato de uma } \\
\text { experiência de pesquisa de } \\
\text { mestrado. }\end{array}$ & $\begin{array}{l}\text { ALVES, Davis } \\
\text { Frederico da Silva }\end{array}$ & $\begin{array}{l}\text { Tecnologias } \\
\text { Comunicacionais e } \\
\text { Informacionais; } \\
\text { Ensino de Funções; } \\
\text { Educação } \\
\text { Relato de uma experiência de } \\
\text { atividade investigativa }\end{array}$ \\
\hline V EMEM & $\begin{array}{l}\text { ALMEIDA, Flávia } \\
\text { Cristina de Faria; } \\
\text { LAUDARES, João } \\
\text { Bosco }\end{array}$ & $\begin{array}{l}\text { Atividade } \\
\text { Investigativa, } \\
\text { Exploração e Busca } \\
\text { por padrões }\end{array}$ \\
\hline
\end{tabular}

Fonte: Elaborado pelas autoras (2018)

No trabalho de Araujo e Melo (2003) no III EMEM, não havia palavras-chaves. Ele defendeu o uso de calculadora como recurso didático nas aulas de matemática. Já a autora FERREIRA (2006) em seu trabalho, no IV EMEM, relatou diversas atividades que utilizam calculadora na Educação de Jovens e Adultos (EJA) que tinham o objetivo de proporcionar aos estudantes um maior entendimento da calculadora, buscando apresentar os alcances e limitações de seu uso. Já Alves e Reis (2009) no V EMEM, o relataram a discussão sobre a utilização de tecnologias, principalmente o uso de calculadora no estudo de funções, com alunos do curso de Licenciatura em Matemática e foi fruto de uma pesquisa do mestrado profissional. Os autores Almeida e Laudares (2009) utilizou a calculadora como metodologia em atividades investigativas almejando uma melhor compreensão dos conteúdos pelos estudantes.

A seguir os trabalhos apresentados na categoria Comunicação Científica:

Tangram - Revista de Educação Matemática, Dourados - MS - v.1, n. 3, pp. 64 - 80 (2018) ISSN: 2595-0967 
Quadro 2: Trabalhos da Categoria Comunicação Científica

\begin{tabular}{|c|c|c|c|}
\hline Evento & Título & Autores & Palavras-Chaves \\
\hline III EMEM & $\begin{array}{l}\text { A presença dos meios } \\
\text { tecnológicos no ensino e } \\
\text { aprendizagem } \\
\text { matemática: professores de } \\
\text { matemática das escolas de } 3^{\circ} \\
\text { e } 4^{\circ} \text { ciclos do ensino } \\
\left.\text { fundamental ( } 5^{\mathrm{a}} \text { a } 8^{\mathrm{a}} \text { séries }\right) \\
\text { públicas e particulares de } \\
\text { belo horizonte usando } \\
\text { tecnologia: quando e como? }\end{array}$ & $\begin{array}{l}\text { GAZIRE, Eliane } \\
\text { Scheid; ARAÚJO, } \\
\text { Denise Alves de; } \\
\text { ROTSEN, Maria } \\
\text { Cristina. LANNES, } \\
\text { Wagner; AUAREK, } \\
\text { Wagner; CUNHA, } \\
\text { Wellington José da. }\end{array}$ & $\begin{array}{l}\text { Tecnologia, } \\
\text { professores de } \\
\text { Matemática, sala de } \\
\text { aula, laboratório de } \\
\text { Matemática }\end{array}$ \\
\hline VI EMEM & $\begin{array}{l}\text { O "jogo da multiplicação": } \\
\text { contribuições para o } \\
\text { processo de ensino e } \\
\text { aprendizagem } \\
\text { matemática para alunos do } 5^{\circ} \\
\text { ano do ensino fundamental }\end{array}$ & $\begin{array}{l}\text { BARROS, Edna } \\
\text { Alvernaz; } \\
\text { FERREIRA, Ana } \\
\text { Rafaela Correia. }\end{array}$ & $\begin{array}{l}\text { Educação } \\
\text { Matemática, Ensino } \\
\text { de multiplicação, } \\
\text { Jogo da } \\
\text { multiplicação. }\end{array}$ \\
\hline VII EMEM & $\begin{array}{l}\text { Uma abordagem geométrica, } \\
\text { com recursos da geometria } \\
\text { dinâmica, para o ensino de } \\
\text { porcentagem }\end{array}$ & MOUTINHO, Ion. & $\begin{array}{l}\text { Geometria } \\
\text { Dinâmica; sistemas } \\
\text { de representação } \\
\text { semiótica; } \\
\text { construção de } \\
\text { conhecimento } \\
\text { matemático; } \\
\text { porcentagem }\end{array}$ \\
\hline
\end{tabular}

Fonte: Elaborado pelas autoras (2018)

A C.C. publicada de Grazire et al (2003) no III EMEM foi fruto de uma pesquisa sobre o uso de tecnologias, principalmente a calculadora, no âmbito do Ensino Fundamental ( $5^{\mathrm{a}}$ a $8^{\mathrm{a}}$ série) com professores da rede particular e pública na cidade de Belo Horizonte onde objetivaram investigar a existência de tecnologias e projetos tecnológicos nestas instituições. Sob outra perspectiva de Barros e Ferreira (2012) no VII EMEM utilizaram a calculadora como metodologia durante um jogo sobre a multiplicação no contexto do Ensino Fundamental com a finalidade de analisar o uso da calculadora e suas contribuições no processo da aprendizagem. Por fim, Mourinho (2015) no VII EMEM tem seu trabalho

Tangram - Revista de Educação Matemática, Dourados - MS - v.1, n. 3, pp. 64 - 80 (2018) ISSN: 2595-0967 
Uso de calculadora: levantamento de trabalhos publicados nos Encontros Mineiros de Educação Matemática

baseado no uso da Calculadora Geométrica de Porcentagens como ferramenta que, de acordo com o autor, permite que os alunos sejam protagonistas do processo de aprendizagem.

\section{CONSIDERAÇÕES FINAIS}

O uso cotidiano das calculadoras na sociedade é bastante frequente e a discussão sobre seu uso, na área da Educação Matemática, já tem certo tempo, bem como a recomendação do seu uso pelos documentos oficiais (PCN - BRASIL, 1997).

A partir do estudo documental realizado nos anais dos EMEM, que contou com a localização dos anais dos EMEM; pesquisa inicial da palavra calculadora nos trabalhos de Relato de Experiência e Comunicação Científica; pesquisa detalhada a partir da leitura dos títulos, resumos, palavra chaves e trechos dos textos que apareciam a palavra calculadora; dos fichamentos de leituras detalhado e sistematização dados dos trabalhos que possuíam foco no uso das calculadoras na sala, concluímos que enquanto divulgação de trabalhos realizados junto à estudantes (relatos de experiência) ou de divulgação de pesquisa (comunicação científica) ainda temos um número bastante reduzido de trabalhos que focam a utilização da calculadora ou seu uso como ferramenta no desenvolvimento de atividades matemáticas em sala de aula. Isso pode ser um reflexo, infelizmente, da sua pouca utilização ou da pouca importância dada ao estudo do seu uso, no âmbito do estado de Minas Gerais, quando pesquisamos um encontro que busca, entre outros objetivos, promover intercâmbio de conhecimentos e divulgar resultados de pesquisas na área de Educação Matemática, no estado. Esperamos que outros trabalhos tenham sido desenvolvidos no período de realização dos EMEM e tenham encontrado como canal de divulgação, outros eventos e publicações e esperamos que novas e mais pesquisas sobre o tema, possam ser divulgadas nos próximos EMEM.

\section{AGRADECIMENTOS}

Agradecemos o apoio da FAPEMIG (Fundação de Amparo à Pesquisa de Minas Gerais) e a Pró Reitoria de Pesquisa de Universidade Federal de Minas Gerais (PRPQ).

Tangram - Revista de Educação Matemática, Dourados - MS - v.1, n. 3, pp. 64 - 80 (2018) ISSN: 2595-0967 
Uso de calculadora: levantamento de trabalhos publicados nos Encontros Mineiros de Educação Matemática

\section{REFERÊNCIAS BIBLIOGRÁFICAS}

ALMEIDA, F. C. F.; LAUDARES, J. B. Relato de uma experiência de atividade investigativa, Encontro Mineiro de Educação Matemática, 5.:2009: Lavras. Anais... Lavras: UFLA, 2009.

ALVES, D. O.; REIS, F. S. Ensino de funções, limites e continuidade em ambientes educacionais informatizados para cursos de introdução ao cálculo: relato de uma experiência de pesquisa de mestrado. Encontro Mineiro de Educação Matemática , 5.:2009: Lavras. Anais eletrônicos... Lavras: UFLA, 2009.

ARAUJO, D. A.; MELO, M. C. R. A calculadora como recurso didático no ensino aprendizagem da matemática: análise das propostas apresentadas nos livros didáticos do ensino fundamental. Encontro Mineiro de Educação Matemática , 3.:2003: Belo Horizonte. Anais eletrônicos... Belo Horizonte: 2003.

BARROS, E. A.; FERREIRA, A. R. C. O "jogo da multiplicação": contribuições para o processo de ensino e aprendizagem em matemática para alunos do $5^{\circ}$ ano do ensino fundamental. Encontro Mineiro de Educação Matemática , 6.2012: Juiz de Fora. Anais eletrônicos... Juiz de Fora: UFJF, 2012.

BORBA, M. C.; SILVA, R. S. R.; GADANIDIS, G. Fases das tecnologias digitais em Educação Matemática: Sala de aula e internet em movimento. 1 ed. Belo Horizonte. Autentica, 2014.

BRASIL. Ministério da Educação e do Desporto. Secretaria de Educação Fundamental. Parâmetros Curriculares Nacionais: matemática. Brasília: MEC/SEF, 1997.

BRASIL. Ministério da Educação. Base Nacional Comum Curricular (BNCC). Educação é a Base. Brasília, MEC/CONSED/UNDIME, 2017.

ENCONTRO MINEIRO DE EDUCAÇÃO MATEMÁTICA, 2.:2000: Belo Horizonte. Anais... Belo Horizonte: UFMG - Escola Fundamental do Centro Pedagógico, 2000. 153p. ENCONTRO MINEIRO DE EDUCAÇÃO MATEMÁTICA, 3.:2003: Belo Horizonte. Anais eletrônicos... Belo Horizonte: UFMG - Escola Fundamental do Centro Pedagógico, 2003.

ENCONTRO MINEIRO DE EDUCAÇÃO MATEMÁTICA, 4.:2006: Diamantina. Anais eletrônicos... Ouro Preto: UFOP, 2006. 1421 p.

Tangram - Revista de Educação Matemática, Dourados - MS - v.1, n. 3, pp. 64 - 80 (2018) ISSN: 2595-0967 
Uso de calculadora: levantamento de trabalhos publicados nos Encontros Mineiros de Educação Matemática

ENCONTRO MINEIRO DE EDUCAÇÃO MATEMÁTICA, 5.:2009: Lavras. Anais eletrônicos... Lavras: UFLA, 2009.

ENCONTRO MINEIRO DE EDUCAÇÃO MATEMÁTICA, 6.:2012: Juiz de Fora. Anais... Juiz de Fora: UFJF, 2012.

ENCONTRO MINEIRO DE EDUCAÇÃO MATEMÁTICA, 7.:2015: São Joao Del Rei. Anais... São Joao Del Rei: UFSJ, 2015.

FERREIRA, A. R. Utilização de calculadora na EJA?. ENCONTRO MINEIRO DE EDUCAÇÃO MATEMÁTICA, 4.:2006: Diamantina. Anais eletrônicos... Ouro Preto: UFOP, 2006.

FIORENTINI, D.; LORENZATO, S. Investigação em educação matemática: percursos teóricos e metodológicos. Campinas-SP: Autores Associados, 2006. (Coleção Formação de Professor).

GAZIRE, E. S.et al. A presença dos meios tecnológicos no ensino e aprendizagem da matemática: professores de matemática das escolas de $3^{\circ}$ e $4^{\circ}$ ciclos do ensino fundamental ( $5^{\mathrm{a}}$ a $8^{\mathrm{a}}$ séries) públicas e particulares de belo horizonte usando tecnologia: quando e como?. ENCONTRO MINEIRO DE EDUCAÇÃO MATEMÁTICA, 3, 2003: Belo Horizonte. Anais eletrônicos... Belo Horizonte, 2003.

LOPES, A. J.; RODRIGUEZ, J. G. Metodologia para o ensino da aritmética: competência numérica no cotidiano. São Paulo: FTD, 2009.

MOUTINHO, I. Uma abordagem geométrica, com recursos da geometria dinâmica, para o ensino de porcentagem. ENCONTRO MINEIRO DE EDUCAÇÃO MATEMÁTICA, 7.:2015: São Joao Del Rei. Anais eletrônicos... São Joao Del Rei: UFSJ, 2015.

SOCIEDADE BRASILEIRA DE EDUCAÇÃO MATEMÁTICA - REGIONAL MINAS GERAIS. Encontro Mineiro de Educação Matemática. Disponível em: $<$ http://www.sbemmg.ufop.br/index.php/8-noticias-gerais/21-viii-emem-encontro-mineirode-educacao-matematica > Acesso em 27 de Fevereiro de 2018.

VAN DE WALLE, J. Matemática no ensino fundamental: formação de professores $e$ aplicação em sala de aula. Tradução de Paulo Henrique Colonese. 6. ed. Porto Alegre: Artmed, 2009.

Recebido:01/03/2018

Aceito:20/06/2018

Tangram - Revista de Educação Matemática, Dourados - MS - v.1, n. 3, pp. 64 - 80 (2018) ISSN: 2595-0967 